\title{
ROL DE ENFERMO CRÓNICO: UNA REFLEXIÓN CRÍTICA DESDE LA PERSPECTIVA DE LOS CUIDADOS ENFERMEROS
}

\author{
Ximena Ibarra Mendoza*, José Siles G onzález**
}

*D octoranda U niversidad de Alicante: Enfermería y Cultura de los Cuidados. Profesora Escuela de Enfermería, U niversidad Arturo Prat, Chile. **DUE. Doctor en H istoria. Licenciado en Pedagogía. Catedrático de Escuela Universitaria. U niversidad de Alicante

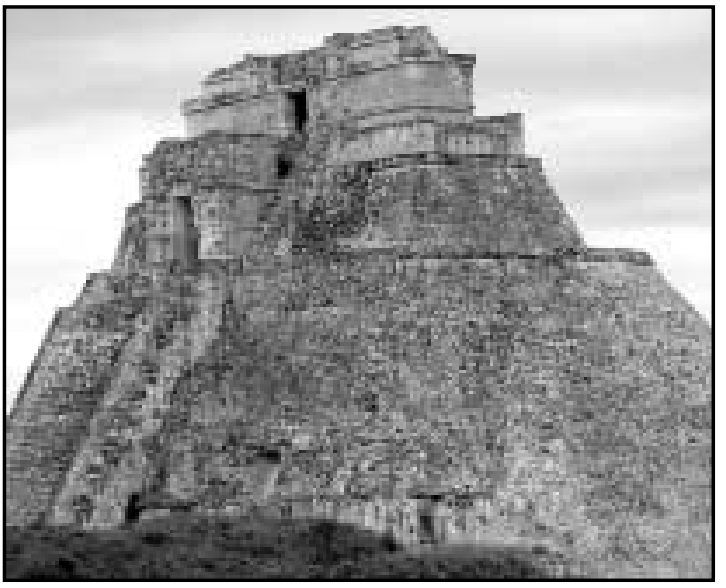

ROLE OF THE CHRONICALLY ILL: A CRITICAL PONDERING FROM THE NURSING CARE POINT OF VIEW

\section{SUMMARY}

? The present review emerges from the need of pondering epistemologically about the pertinence of the nursing care in sight of the experience of living with a chronic illness. It starts from the assumption of the complexity of the reality of the human being (understanding the person as the sum of structures that conform a whole in a dynamic way, influenced by internal and external factors, with complex, dynamics and hard to foresee behaviors). The process of becoming ill involves in itself important changes and disorders in our system, and if we add to this the modifications that must be adopted in the everyday life, it will affect not only the patient, but also all who surround.

The theme was structured by means of an analysis process centered in the symbolic interactionism of Blumer, considering human life mainly as a vast interpretative process in which people, either individually or collectively, guide themselves trough the definition of the objects, events and situations in which they are, interacting the physiological plane (senses) with the intellectual and historical (previous knowledge and experiences).

Those who receive the nursing care are individuals who struggle constantly to "give a sense" to the world that surrounds them. However, they differ one from another in the way in which they give sense to the events affecting them.

The nursing care professional must not only contribute to the treatment of the disease, but also help the person to deal with it in total coherence with the world.

Key words: experience of living; chronic illness; symbolic interactionism; nursing care.

\section{RESUMEN}

Esta revisión surge de la necesidad de reflexionar epistemológicamente sobre la pertinencia de los cuidados enfermeros ante la experiencia de vivir con una enfermedad crónica.

Se parte del supuesto que la realidad del ser humano es compleja (entendiendo a la persona como conjunto de estructuras que forman un todo de manera dinámica, influenciado por factores internos y externos, con comportamientos complejos, dinámicos y difíciles de predecir). El proceso de enfermar por sí solo supone importantes cambios y desordenes en nuestro sistema, y si a ello, le agregamos las modificaciones que debe adoptar en las actividades de la vida diaria, afectará no sólo a la persona que lo padece, sino a todos los que le rodean. 
El tema se estructuro mediante un proceso de análisis centrado en el interaccionismo simbólico de Blumer, considerando la vida humana principalmente como un vasto proceso interpretativo en el que las personas, individual y colectivamente, se guían mediante la definición de los objetos, eventos y situaciones en que se encuentran, interactuando el plano fisiológico (sentidos) con el intelectivo e histórico (conocimientos y experiencias previas).

Quienes reciben los cuidados de enfermería son individuos que luchan constantemente para "dar un sentido" al mundo que les rodea. Sin embargo difieren uno de otro en la forma en que dan sentido a los acontecimientos que les afectan

El profesional de enfermería no sólo debe contribuir en el tratamiento de estas enfermedades, sino ayudar a que la persona conviva con ella en total coherencia con el mundo.

Palabras clave: experiencia de vivir; enfermedad crónica; interaccionismo simbólico; cuidados enfermeros.

\section{INTRODUCCIÓN:}

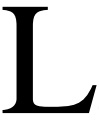

a enfermedad crónica implica, que numerosos enfermos sobrellevan sus males durante décadas, demandando una atención permanente y adecuada. La prolongación de los síntomas a largo plazo perjudica su capacidad para seguir con actividades significativas y rutinarias. Su tratamiento médico suele tener una eficacia limitada, y contribuye a veces al malestar físico y psicológico tanto del paciente como su familia. Un proceso crónico hace impacto típicamente en la percepción que la persona tiene de su cuerpo, su orientación en el tiempo y el espacio, su capacidad para predecir el curso y los acontecimientos de la vida, su autoestima, y sus sentimientos de motivación y dominio personal. Esto supone la necesidad de una atención integral que considere al individuo, familia y su entorno social frente a un problema de salud como una enfermedad degenerativa, sin olvidar que es indispensable el desarrollo y capacidad de armonizar, para y en la sociedad. Quienes reciben los cuidados de enfermería son individuos que luchan constantemente para "dar un sentido" al mundo que les rodea. Sin embargo difieren una de otra en la forma en que dan sentido a los acontecimientos que les afectan

En consideración a esto, se plantea la presente revisión con el objetivo de reflexionar epistemológicamente sobre la pertinencia de los cuidados enfermeros ante la experiencia de vivir con una enfermedad crónica. El tema se estructuro mediante un proceso de análisis centrado en el interaccionismo simbólico de Blumer, considerando la vida humana principalmente como un vasto proceso interpretativo en el que las personas, individual y colectivamente, se guían mediante la definición de los objetos, eventos y situaciones en que se encuentran, interactuando el plano fisiológico (sentidos) con el intelectivo e histórico (conocimientos y experiencias previas).

\section{Rol de enfermo:}

El concepto de sick role de Parsons (1951) se basa en el supuesto de que estar enfermo no es una elección consciente y deliberada, aunque una enfermedad pueda producirse como consecuencia de una exposición intencionada a una infección o una lesión. Parsons advierte, sin embargo, que algunas personas pueden verse atraídas por el rol de enfermo en cuanto que la persona enferma no puede desarrollar sus tareas normales. El estar enfermo, dice Parsons, no es sólo experimentar la condición física de enfermedad; más bien constituye un rol social porque implica conductas basadas en expectativas institucionales, y este rol social se ve reforzado por las normas de la sociedad que corresponden a esas expectativas.

Sin embargo, Mechanic (1962a), por ejemplo, sugiere que la edad, el sexo, la importancia de los roles sociales y las respuestas aprendidas frente a los síntomas varían entre los individuos.

Gordon (1966) por su parte, en una investigación realizada en Nueva York, encontró que, cuando se creía que el pronóstico era grave e incierto, las conductas anticipadas coincidían generalmente con la descripción de Parsons; sin embargo, cuando el pronóstico era conocido pero no era grave, la noción de un "rol disminuido" surgió de los datos de Gordon, y este rol exigía el ejercicio de las responsabilidades normales y rechazaba las exenciones a pesar de la enfermedad. 
Twaddle (1969) mostró al menos siete configuraciones del rol de enfermo, en un estudio de matrimonios de edad madura en Rhode Island. La configuración exacta de los roles de enfermo alternativos descubiertos por Twaddle dependía en parte de los valores culturales y de si una persona se definía como "enferma". No sólo existían diferentes definiciones personales respecto a "estar enfermo", sino que no todos los entrevistados decían que esperaban recuperarse, y no todos colaboraban con el médico.

Una segunda crítica al concepto de Parsons, es que sólo parece aplicarse a las enfermedades agudas, que por naturaleza son temporales, normalmente reconocibles por no profesionales y fácilmente superables con ayuda médica. Sin embargo, las enfermedades crónicas como el cáncer o las afecciones cardiovasculares no son temporales, y en muchas ocasiones no es posible que el paciente se ponga bien según el modelo de Parsons, independientemente del deseo que tenga el paciente de colaborar con el médico.

Investigaciones sobre pacientes con trastornos crónicos (Kassebaum y Baumann 1965; Radley 1994) señalan que su percepción del rol de enfermo es diferente de la de los enfermos agudos. Los pacientes crónicos tienen que enfrentarse a la imposibilidad de volver a desempeñar roles de forma normal, o de ajustar sus actividades a un trastorno permanente. Sin embargo, en una reconsideración del rol del enfermo, Parsons (1975) sostiene que incluso si el objetivo de la recuperación completa no es factible, muchas enfermedades crónicas pueden ser "manejadas" para que el paciente pueda mantener un funcionamiento fisiológico y social bastante normal.

Así pues, se puede comprender que un estado esencialmente malsano no siempre es identificado como enfermedad, cuando las personas afectadas pueden funcionar normalmente y la presencia del trastorno no afecta el ritmo normal de la vida cotidiana. Por consiguiente, las opiniones sobre lo que es una enfermedad o una conducta desviada son relativas y no pueden separarse de las situaciones sociales en las que vive la gente.

Eliot Freidson (1970a), indica que la clave para distinguir entre los roles de enfermo es la noción de la legitimidad, que implican una desviación mayor o menor. (1) La legitimidad condicional, en la que los desviados tienen una exención temporal de sus obligaciones habituales y consiguen algunos privilegios adicionales siempre que busquen ayuda para librarse de su desviación; un catarro sería una desviación menor y una neumonía, una grave dentro de esta categoría. (2) La legitimidad incondicional, en la que los desviados tienen una exención permanente de sus obligaciones normales y se les conceden privilegios adicionales a la luz de la grave naturaleza de su desviación; el cáncer Terminal entraría en esta categoría. (3) La ilegitimidad, en la que los desviados están exentos de algunas de sus obligaciones en virtud de su desviación, de la cual no son responsables en términos técnicos; sin embargo, se les conceden pocos privilegios (si es que los hay) y asumen impedimentos tales como estigmas; un tartamudeo sería una desviación leve y una epilepsia, una grave.

El sistema de clasificación de Freidson supone que hay diferentes consecuencias para el individuo y que su tratamiento por parte de otras personas depende de la etiqueta de definición aplicada al trastorno por otros. El modelo de Freidson tiene en cuenta los aspectos problemáticos de la enfermedad en relación con las situaciones sociales.

El concepto de Freidson, sin embargo, es puramente teórico y no ha sido sometido a pruebas exhaustivas. Por tanto, queda por ver si será capaz de explicar las variaciones en la conducta de enfermedad. Aunque este modelo tiene su utilidad en la clasificación de la conducta de enfermedad, no consigue explicar las diferencias en la forma en que las personas se definen como "enfermas" y necesitadas de atención médica profesional. El mérito del modelo de Freidson reside en que va más allá del concepto de Parsons, ya que describe diferentes tipos de enfermedad y señala que la misma es una etiqueta creada por la sociedad.

No obstante, cuando una persona está enferma no es la de siempre. Es diferente (anormal) en una manera negativa que la mayoría de las personas preferiría evitar como evitan ir a la cárcel, por ejemplo. Los enfermos no sólo se sienten mal, sino que pueden estar también físicamente discapacitados. Asimismo, pueden experimentar disfunciones mentales. Según Eric Cassell (1985) las personas con enfermedades físicas graves pierden su pers- 
pectiva y son incapaces de pensar desde más de un punto de vista. Su capacidad de razonar y tomar decisiones puede verse alterada, y pueden llegar a depender de los médicos y de otras personas para su cuidado. También se pueden volver sobre sí mismos y mostrarse infantiles hasta el punto de pensar exclusivamente en ellos e ignorar el mundo exterior.

Kathy Charmaz $(1983,1991)$ sugiere que los enfermos crónicos también experimentan un sentido negativo del yo porque su enfermedad limita sus actividades, les aísla de los demás, les desacredita porque reduce su sentido de valor personal, y hacen que sean una carga para otros. De hecho, algunas personas pueden sentirse estigmatizadas como consecuencia de su enfermedad, como se ve en casos de personas con impedimentos físicos (Zola 1982), la enfermedad de Parkinson (Lefton, 1984), Epilepsia (Scambler y Hopkins 1986) y otros problemas como la Diabetes, enfermedades renales en estados avanzados, y la esclerosis múltiple (Roth y Conrad 1987). Efectos como estos parecen colocar a los enfermos en una situación de desviación respecto a su sentido del yo, así como en las relaciones con otras personas y en el desempeño de su papel social.

Como explican Peter Freund y Meredith McGuire (1999): "la enfermedad es triste porque se siente como una amenaza al orden y a los significados a través de los cuales la gente da sentido a su vida". Lo que quieren decir Freund y McGuire aquí, es que la enfermedad trastorna la rutina diaria, propicia diferentes grados de sufrimiento y perjudica la capacidad de programar el futuro y controlar las actividades. Estar enfermo es tener envidia de los que están bien o incluso del pasado sano; se espera poder volver a disfrutar de buena salud. Esto puede ser imposible si uno tiene una enfermedad crónica.

Una enfermedad crónica puede desarrollarse lentamente, dura mucho tiempo y normalmente llega a ser incurable. Las enfermedades crónicas se desarrollan dentro del organismo en lugar de ser transmitidas de una persona a otra y suelen ser asociadas con influencias genéticas, medioambientales y estilos de vida. Si la enfermedad crónica es peligrosa para la vida (ej. Cáncer, diabetes, enfermedades cardiovasculares) es probable que la per- sona afectada acabe muriéndose de ellas.

Independientemente de si la dolencia es aguda o crónica, cuando las personas la sufren la sensación de competencia personal puede verse disminuida.

Freund y McGuire (1999) señalan que una enfermedad puede ser particularmente dañina para el concepto de sí mismo cuando se experimenta como algo abrumador, impredecible e incontrolable porque paraliza la capacidad de actuar y de llevar una vida normal. Las personas muy enfermas sienten con frecuencia una sensación de alienación o separación física de sus cuerpos porque les parece que ya no funcionan adecuadamente o de forma normal (Ezzy 2000; Freund y McGuire 1999; Kelly y Field 1996; Leventhal 1975). Los enfermos a menudo se retiran del mundo porque se sienten mal y no son capaces de mantener sus relaciones sociales normales.

Las personas afectadas por enfermedades crónicas, y sobre todo los discapacitados físicos, tienen que enfrentarse a problemas adicionales de movilidad, una imagen corporal negativa y el estigma social (S. Bell 2000; P. Brown 1995; Bury 1991, 1997, 2000; Ferraro, Farmer y Wybraniec 1997; Radley 1989, 1993, 1994; Roth y Conrad 1987; Schieman y Turner 1998; G. Williams 1999, 2000; Yoshida 1993). Por consiguiente, Zola $(1982,1989)$ indica que los problemas de personas con una discapacidad física no son exclusivamente médicos, sino que incluyen factores sociales, económicos y de actitud, entre otros.

Charmaz (1983, 1991), en un estudio sobre enfermos crónicos en el norte de California, encontró que estos individuos experimentan con frecuencia una imagen deteriorada de sí mismos y no son capaces de asumir una nueva que tenga el mismo valor que la anterior. Como consecuencia de su enfermedad o discapacidad tienen vidas limitadas, están socialmente aislados, se les considera personas con capacidades por debajo de lo normal, y sienten que son una carga para otras personas. Todos estos factores se combinan para reducir el sentido de autoestima del enfermo, a no ser que se pueda encontrar otro medio de satisfacción.

Como concluye Bury (2000): "Los cambios en el organismo interactúan con la sociedad en contextos culturales y estructurales que van más allá 


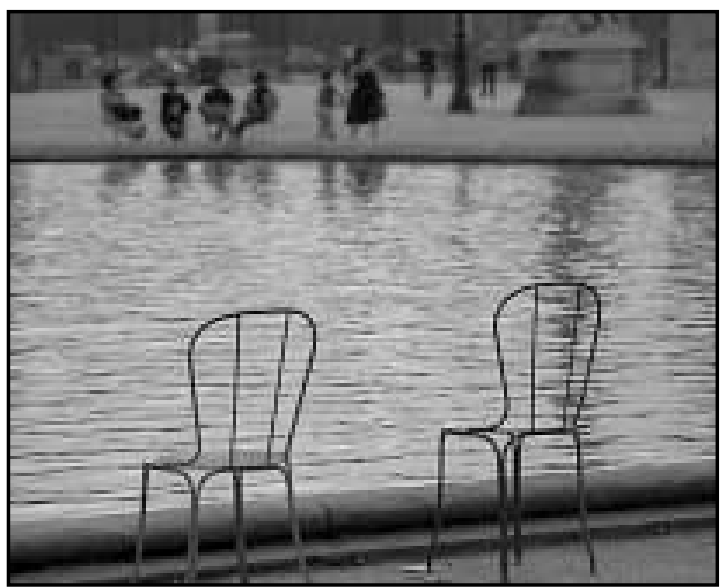

de las consecuencias prácticas de su situación, para entrar en el ámbito de cuestiones como el aspecto físico, el funcionamiento social y su propia identidad".

Anselm Strauss (1975; Strauss y Corbin 1988) explica que la misión principal de una persona con una enfermedad crónica no es sólo mantenerse viva o controlar sus síntomas, sino vivir una vida lo más normal posible. Una enfermedad que dura toda la vida, en opinión de Strauss, requiere el trabajo continuo de controlar su curso, de manejar sus síntomas y convivir con la discapacidad. En este contexto, el rol de enfermo asumido por la persona es una condición permanente.

\section{En un contexto epistemológico.}

El tratamiento de estas enfermedades supone la consideración de varios factores causales, los cuales no son todos de origen biológico. Los factores sociales y psicológicos no sólo influyen en si una persona se convierte en enfermo o no, sino también en la forma, desarrollo e intensidad de los síntomas.

Goffman (1959), señala que las personas viven en ámbitos de relaciones sociales en las cuales actúan de forma verbal y no verbal donde los individuos expresan su opinión sobre una situación, sobre los demás participantes y, particularmente, sobre sí mismas, es decir, "la percepción del yo" que se proyecta a las otros. Sin embargo, el mismo autor señala, que esta percepción del yo sólo viene prestada por la sociedad y puede ser retirada si la persona se comporta de forma errónea.
Cuando aparecen situaciones nuevas ó alternativas y la conducta habitual se distorsiona, cabe esperar una sensación de desorganización e incertidumbre. La capacidad del individuo para sobrellevar una situación límite tiene relación muy estrecha con las experiencias de socialización, que le habrán enseñado la forma en que debe afrontar las nuevas situaciones

El trabajo de W. Thomas Volkart (1951), aporta dos conceptos importantes frente a esta cuestión, la primera es que una misma situación de crisis no produce el mismo efecto en las personas de manera uniforme. Y en segundo lugar, explica que el ajuste y el control de una situación de crisis es el resultado de la capacidad del individuo de comparar una situación actual con situaciones similares del pasado y adaptar su juicio y su modo de actuar sobre la base de la experiencia anterior. El resultado de una situación particular depende, por tanto, de la definición por el individuo de una situación y de la forma en que ese individuo acepta la situación.

Para el caso de las enfermedades crónicas, cabe también esperar que las personas que la padecen, tengan diferentes interpretaciones de la situación que están viviendo, aunque finalmente es la forma en que perciben las tensiones y los conflictos en sus roles, áreas, relaciones personales y otros aspectos de su situación vital lo que les da el sentido a sus vidas.

\section{El cuidado enfermero desde la perspectiva del Interaccionismo simbólico.}

La conducta de enfermedad es una respuesta cultural y socialmente aprendida. Una persona responde ante los síntomas de acuerdo con su definición de la situación. Esta definición puede estar influenciada por las definiciones de otros, pero principalmente está determinada por el aprendizaje, la socialización y por experiencias pasadas como los que proporcionan sus antecedentes u orígenes culturales y sociales.

Desde el punto de vista del paradigma interpretativo simbólico, los profesionales de enfermería actúan como facilitadores-cooperadores con el sujeto (paciente) respecto de la optimización de su proceso salud enfermedad. La naturaleza del conocimiento respecto de dicho proceso no es objetivo, 
sino subjetivo y la acción de enfermería se desarrolla en un clima de interacción y constante intersubjetividad, teniendo en cuenta la realidad específica del paciente. (Siles, J. y García, E. 1995).

Por tanto, se podría decir que el interaccionismo simbólico está basado en tres suposiciones básicas. Primero, la realidad social como la percibimos, conocemos y entendemos, es una producción social. Los individuos que interactúan producen y determinan sus propias definiciones de las situaciones. Segundo, se da por hecho que los seres humanos son capaces de entablar un comportamiento pensado y autorreflexivo. Son capaces de dar forma y de guiar tanto su propio comportamiento como el de los demás. Tercero, en el curso de adoptar su propio punto de vista y adecuarlo al comportamiento de los demás, los seres humanos interactúan entre sí. La interacción es simbólica porque incluye la manipulación de símbolos, palabras, significados y lenguajes. El significado de los objetos está en las acciones que llevan a cabo los seres humanos en relación con ellos. (Blumer, 1969)

Quienes reciben los cuidados de enfermería son individuos que luchan constantemente para "dar un sentido" al mundo que les rodea. Las personas son, por encima de todo, "dadores de significado" de las situaciones en las que se encuentran. Sin embargo difieren una de otra en la forma en que dan sentido a los acontecimientos que les afectan. Dentro del contexto de los cuidados de la salud es particularmente probable que, las personas intenten comprender la causa y naturaleza de su estado de enfermedad así como su probable duración.

\section{Conclusiones.}

La enfermedad, como alteración de vida, de rutina, es algo a olvidar. El enfermo no es marginal a esto, intenta por todos los medios olvidar su enfermedad, marginarla, para liberarse y volver al mundo de la salud, transformado en un sano amnésico.

El profesional de enfermería no sólo debe contribuir en el tratamiento de estas enfermedades, sino ayudar a que la persona conviva con ella en total coherencia con el mundo.

Parte de la tarea de enfermería es, por lo tanto, intentar entrar en el mundo subjetivo del paciente para ver las cosas como él. Sólo de este modo puede la enfermera hacer una valoración exacta de las necesidades del individuo y planificar el cuidado holisticamente.

\section{Propuestas y recomendaciones.}

- Es necesario que los profesionales de enfermería comprendan cómo los pacientes construyen e interpretan su enfermedad. Si se logra conocer la forma en que los individuos responden ante una problemática y las razones por las cuales lo hacen de esa forma, quizá entonces se puedan modificar sus acciones en forma más idónea.

- Estudiar las enfermedades crónicas desde la perspectiva de la investigación cualitativa ayudaría a comprender el significado de vivir con ella y con un cúmulo de fenómenos interrelacionados a ésta, los que se caracterizan por ser: prolongados; de pronóstico incierto; y muchos de ellos de naturaleza inherentemente episódico, además de adaptarse a los efectos secundarios de los procedimientos médicos, los que podrían perturbar de manera desproporcionada la vida tanto de los pacientes como la de sus familias, añadiendo a esta situación el hecho de que su tratamiento y/o rehabilitación es relativamente costoso.

\section{BIBLIOGRAFÍA}

- Bell, SE. 2000. "Experiencing illness in/and narrative", pp. 184-199 in Handbook of medical sociology, 5th ed., C. Bird, P. Conrad, and A. Fremont (eds.). Upper Saddle River, NJ: Prentice Hall.

- Blumer, H. (1969). Symbolic Interactionism: Perspective and method. Englewood Cliffs: Prentice Hall

- Brown, P. 1995. "Naming and framing: The social construction of diagnosis and illness". Journal of Health and Social Behavior, extra issue: 34-52.

- Bury, M. 1991. "The sociology of chronic illness: A review of research and prospect". Sociology of Health and Illness, 13:451-468.

- 1997. Health and illness in a changing society. London: Routledge.

- 2000. "On chronic illness and disability", pp. 173-183 in Handbook of medical sociology, 5th ed., C. Bird, P. Conrad, and A. Fremont (eds.). Upper Saddle River, NJ. Prentice Hall. - Cassell, E J. 1985. Talking with patients, Vol. 2. Cambridge, Mass.: MIT Press.

- Charmaz, K.1983. "Loss of self: A fundamental form of suffering in the chronically ill". Sociology of Health and Illness, 5:168-195.

- 1991. Good days, bad days: The self in chronic illness and time. New Brunswick, N.J.: Rutgers University Press.

- Ezzy, D. 2000. "Illness narratives: Time, hope and HIV". Social Science and Medicine, 50:605-617. 
- Ferraro, KF; Farmer, MM and Wybraniec, J A. 1997. "Health trajectories: Long-term dynamics among black and white adults". Journal of Health and Social Behavior, 38:38-54.

- Freidson, E. 1970a. Profession of medicine. New York: Dodd, Mead.

- Freund, PES. and McGuire, MB.. 1999. Health, illness, and the social body, 3rd ed. Englewood Cliffs, N.J.: Prentice Hall. - Goffman, E. 1959. The presentation of self in everyday life. New York: Anchor.

- Gordon, G. 1966. Role theory and illness. New Haven, Conn.: College and University Press.

- Kassebaum, G., and Baumann, B.. 1965. "Dimensions of the sick role in chronic illness". Journal of Health and Social Behavior, 6:16-27.

- Kelly, MP. and Field, D. 1996. "Medical sociology, chronic illness and the body". Sociology of Health and Illness, 18:241-257

- Lefton, M. 1984. "Chronic disease and applied sociology: An essay in personalized sociology". Sociological Inquiry, 54:466-476.

- Leventhal, H. 1975. "The consequences of personalization during illness and treatment: An information-processing model", pp. 119-162 in Humanizing health care, J. Howard and A. Strauss (eds.). New York: Wiley-Interscience.

- Mechanic, D. 1962a. "The concept of illness behavior". Journal of Chronic Diseases, 15:189-194.

- Parsons, T. 1951. The social system. Glencoe, III.: The Free Press.

- 1975. "The sick role and role of the physician reconsidered". Milbank Memorial Fund Quarterly, 53:257-278.

- Radley, Alan. 1989. "Style, discourse and constraint in adjustment to chronic illness". Sociology of Health and Illness, 11:230-252.

- (ed). 1993. Worlds of illness: Biographical and cultural perspectives on health and disease. London: Routledge.
- 1994. Making sense of illness: The social psychology of health and disease. London: Sage.

- Roth, J and Conrad, P (eds.) 1987. The experience and management of chronic illness. Newbury Park, Calf.: Sage

- Scambler, G and Hopkins, A 1986. "Being epileptic: Coming to terms with stigma". Sociology of Health and Illness, 8:2643.

- Schieman, S. and Turner, H.A 1998. "Age, disability, and the sense of mastery". Journal of Health and Social Behavior, 39: 169-186.

- Siles, J. y García, E. (1995). Las características de los paradigmas y su adecuación a la investigación de Enfermería. Enfermería Científica. Num. 160-161. Julio-Agosto.

- Strauss, A. 1975. Chronic illness and the quality of life. St. Louis: C. V. Mosby.

- Strauss, A and Corbin, J M.. 1988. Shaping a new health care system. San Francisco: Jossey-Bass.

- Twaddle, A. 1969. "Health decisions and sick role variations: An exploration". Journal of Health and Social Behavior, 10:105-114.

- Volkart, E. H. (ed.). 1951. Social behavior and personality, New York: Social Science Research Council.

- Williams, SJ. 1999. "Is anybody there? Critical realism, chronic illness and the disability debate". Sociology of Health and Illness, 21:797-819.

- 2000. "Chronic illness as biographical disruption or biographical disruption as chronic illness". Sociology of Health and Illness, 22:40-67.

- Yoshida, KK. 1993. "Reshaping of self: A pendular reconstruction of self and identity among adults with traumatic spinal cord injury". Sociology of Health and Illness, 15:217-245.

- Zola, I. 1982. Missing pieces: A chronicle of living with a disability. Philadelphia: Temple University Press.

- 1989. "Toward the necessary universalizing of a disability policy”. Milbank Quarterly, 67, Supp. 2:401-428.

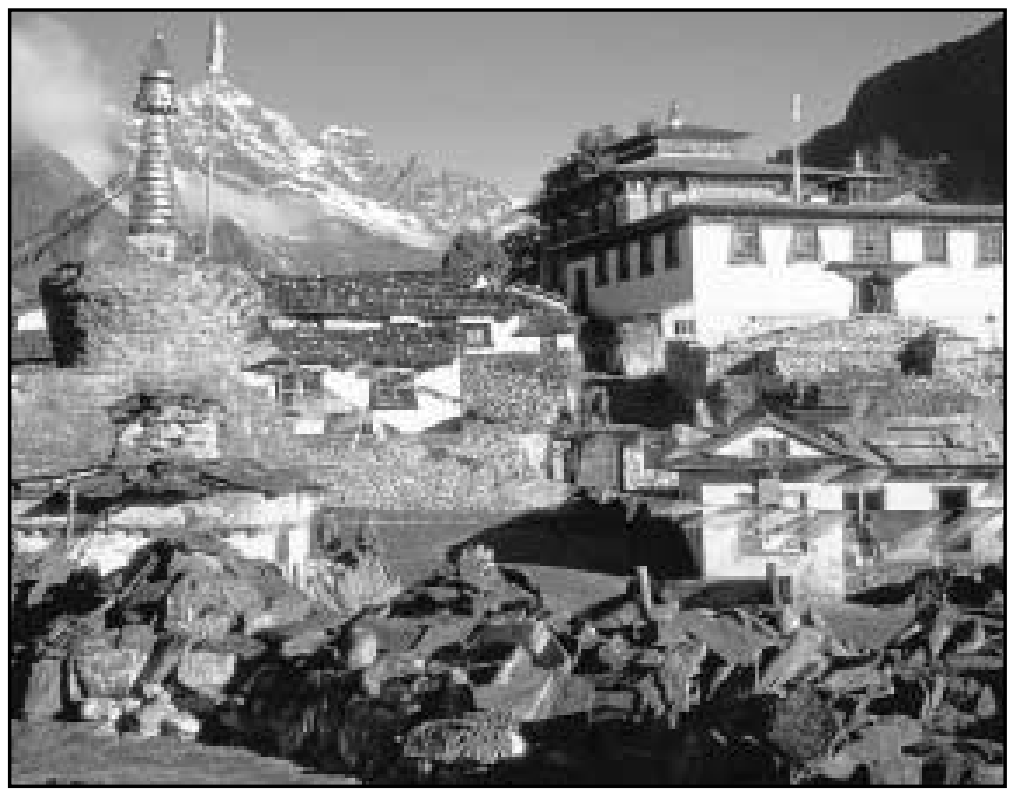

